\title{
ANOTHER VIEW OF BIPARTITE RAMSEY NUMBERS
}

\author{
Zhenming Bi, Gary Chartrand \\ AND \\ Ping ZHANG \\ Department of Mathematics \\ Western Michigan University \\ Kalamazoo, MI 49008, USA \\ e-mail: ping.zhang@wmich.edu
}

\begin{abstract}
For bipartite graphs $F$ and $H$ and a positive integer $s$, the $s$-bipartite Ramsey number $B R_{s}(F, H)$ of $F$ and $H$ is the smallest integer $t$ with $t \geq s$ such that every red-blue coloring of $K_{s, t}$ results in a red $F$ or a blue $H$. We evaluate this number for all positive integers $s$ when $F=K_{2,2}$ and $H \in\left\{K_{2,3}, K_{3,3}\right\}$.
\end{abstract}

Keywords: Ramsey number, bipartite Ramsey number, $s$-bipartite Ramsey number.

2010 Mathematics Subject Classification: 05C35, 05C55.

\section{REFERENCES}

[1] E. Andrews, G. Chartrand, C. Lumduanhom and P. Zhang, Stars and their kRamsey numbers, Graphs Combin. 33 (2017) 257-274. doi:10.1007/s00373-017-1756-9

[2] L.W. Beineke and A.J. Schwenk, On a bipartite form of the Ramsey problem, in: Proceedings of the Fifth British Combinatorial Conference (Univ. Aberdeen, Aberdeen, 1975) 17-22.

[3] Z. Bi, G. Chartrand and P. Zhang, Party problems and Ramsey numbers, preprint.

[4] G. Chartrand and P. Zhang, Chromatic Graph Theory (Chapman \& Hall/CRC Press, Boca Raton, FL, 2009).

[5] T.P. Kirkman, On a problem in combinatorics, Cambridge and Dublin Math. J. 2 (1847) 191-204. 